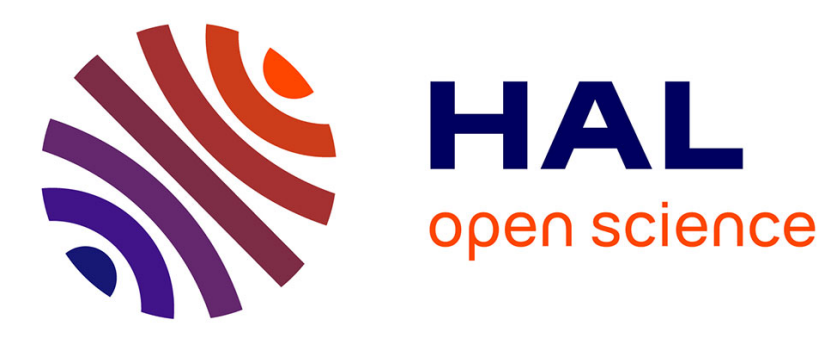

\title{
MRI CONTRAST ENHANCEMENT OF MAGNETIZATION PREPARED STEADY STATE SEQUENCE: AN OPTIMAL CONTROL FRAMEWORK
}

Benoît Vernier, Eric Van Reeth, Frank Pilleul, Oliver Beuf, Hélène Ratiney

\section{To cite this version:}

Benoît Vernier, Eric Van Reeth, Frank Pilleul, Oliver Beuf, Hélène Ratiney. MRI CONTRAST ENHANCEMENT OF MAGNETIZATION PREPARED STEADY STATE SEQUENCE: AN OPTIMAL CONTROL FRAMEWORK. ISBI, Apr 2021, NICE, France. hal-03213076

\section{HAL Id: hal-03213076 https://hal.science/hal-03213076}

Submitted on 30 Apr 2021

HAL is a multi-disciplinary open access archive for the deposit and dissemination of scientific research documents, whether they are published or not. The documents may come from teaching and research institutions in France or abroad, or from public or private research centers.
L'archive ouverte pluridisciplinaire HAL, est destinée au dépôt et à la diffusion de documents scientifiques de niveau recherche, publiés ou non, émanant des établissements d'enseignement et de recherche français ou étrangers, des laboratoires publics ou privés. 


\title{
MRI CONTRAST ENHANCEMENT OF MAGNETIZATION PREPARED STEADY STATE SEQUENCE: AN OPTIMAL CONTROL FRAMEWORK
}

\author{
Benô̂t Vernier ${ }^{1,2}$, Eric Van Reeth ${ }^{1,3}$, Frank Pilleul ${ }^{1,4}$, Oliver Beuf ${ }^{1}$, Hélène Ratiney ${ }^{1}$ \\ ${ }^{1}$ CREATIS, Université de Lyon, INSA Lyon, UCBL Lyon 1, UJM Saint Etienne, Unité CNRS UMR 5220, INSERM U1206, \\ F69621 Lyon, France \\ ${ }^{2}$ SIEMENS Healthineers, 93210 Saint-Denis, France, ${ }^{3} \mathrm{CPE}$ Lyon, France, ${ }^{4}$ Centre de lutte contre le cancer Léon Bérard, \\ Department of Radiology, Lyon, France
}

\begin{abstract}
The present work investigates the possibilities of enhancing MRI contrast between two target tissues in a threedimensional Magnetization-Prepared Rapid Gradient-Echo (MP-RAGE) based on relaxation time's differences. The benefit lies in the adaptation of the GRAPE algorithm to the optimization of a magnetization preparation in a cyclic sequence without full recovery between each cycle. This numerical approach allows optimizing a magnetization preparation of an arbitrary number of radio frequency pulses to enhance contrast, taking in account the establishment of a steady state in the longitudinal component of the magnetization. In vivo validation on rat brain is performed at 11.7T and shows the benefit of an optimized $\mathrm{T}_{2}$ Prep-IR compared to a simple inversion to enhance contrast between white and gray matter.
\end{abstract}

Index Terms- GRAPE, Optimal Control, MP-RAGE

\section{INTRODUCTION}

Since the early work of Mugler et al. [1], MP-RAGE is one of the most common sequence for $\mathrm{T}_{1}$ weighted MRI of the brain. This sequence allows high spatial resolution and high contrast images in times adapted to a clinical context. A MPRAGE sequence is typically divided into cycles (or segments). Each cycle contains three periods: (1) a time devoted to partial magnetization recovery, (2) a magnetization preparation period for contrast control, (3) several data acquisitions with a short time of repetition (TR) and low flip angle using a spoiled gradient echo scheme. Each cycle fills a fraction of the $\mathrm{k}$-space. The establishment of the longitudinal steady state without any contribution of the transverse magnetization (due to the gradient echo excitation pulses) supposes to select coherent transverse pathway. In practice disrupting transverse coherence is achieved by incrementing the RF pulse phase and using gradient spoiler [2].

The optimization of the sequence remains a difficult numerical problem due to coupling between cycles. Mugler and al. [3] have developed a solution in the simple case of a single RF pulse preparation. However, improvements can be made to increase the types and/or quality of contrast achievable. For example, one decade ago, Marques and al. [4] proposed an improvement of this sequence with the MP2RAGE which has two periods of acquisitions per cycle. In this context, our approach is to investigate the possibilities of enhancing contrast by modeling an arbitrary preparation inside the MP-RAGE. Our work is based on the recent optimal control framework proposed for the design of a preparation to optimize MRI contrast based on the relaxations differences [5-7].

This paper presents a novel use of the GRAPE algorithm in order to optimize a complex preparation within the establishment of a longitudinal steady state. It generalizes previous optimal control based algorithm in which full magnetization recovery was required. In addition, it presents the contrast improvement that can provide an optimized $\mathrm{T}_{2}$ Prep-IR compared to a standard MP-RAGE on rat brain.

\section{METHOD}

\subsection{Modeling of a $T_{1} w$ MP-RAGE}

For a simple inversion-recovery preparation, the cyclic nature of the sequence with no-full recovery of the magnetization between each cycle leads to a stationary state quite easy to compute. The evolution of the longitudinal magnetization within one cycle, $M_{z, j=1 . .4}$, is described below: Cycle $n^{\circ} \mathrm{k}$

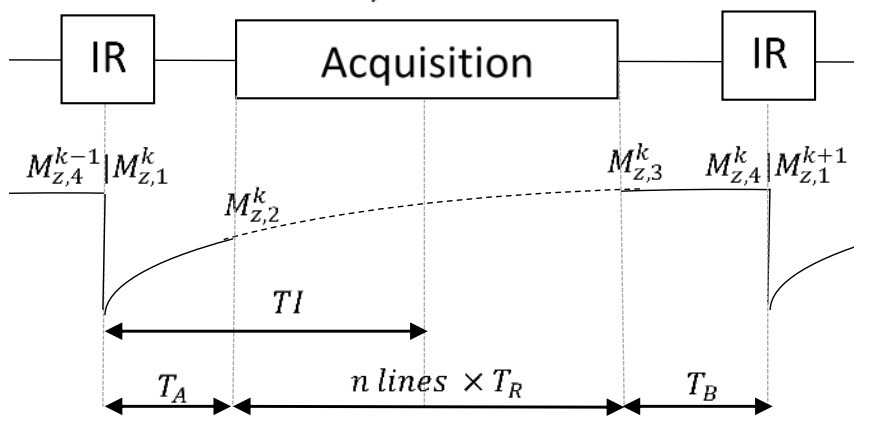

Fig. 1. Schematic diagram of the $T_{1}$ MP-RAGE sequence 
$M_{z, 1}{ }^{k+1}=-e f f \cdot M_{z, 4}{ }^{k}$

$M_{z, 2}{ }^{k+1}=M_{0}(1-E A)+M_{z, 1}{ }^{k+1} E A$

$M_{z, 3}{ }^{k+1}=M_{0} S_{1}\left(1-K_{1}^{n}\right)+K_{1}^{n} M_{z, 2}{ }^{k+1}$

$M_{z, 4}{ }^{k+1}=M_{0}(1-E B)+M_{z, 3}{ }^{k+1} E B$

With: $E A=e^{-\frac{T A}{T_{1}}}, E B=e^{-\frac{T B}{T_{1}}}, K_{1}=\cos (\alpha) e^{-\frac{T_{R}}{T 1}}, \alpha:$ the flip angle (degrees), $T_{R}$ : the time of repetition of the gradient echo scheme (ms), $n$ the number of lines encoded per cycle, eff : the efficacy of the inversion and $M_{0} S_{1}$ : the steady state associated to the gradient echo acquisition sequence, $S_{1}=$ $\left(1-e^{-\frac{T_{R}}{T 1}}\right) /\left(1-\cos (\alpha) e^{-\frac{T_{R}}{T 1}}\right)$.

By iteratively inserting the first equations in the last one, we find:

$\boldsymbol{M}_{z, \mathbf{4}}{ }^{\boldsymbol{k}+\mathbf{1}}=M_{0}(1-E B)+M_{0} S_{1}\left(1-K_{1}^{n}\right) E B+$ $M_{0} K_{1}^{n} E B(1-E A)-e f f . K_{1}^{n} E B E A \boldsymbol{M}_{z, \mathbf{4}}{ }^{k}$

Then writing $M_{z, 4}{ }^{k+1}=M_{z, 4}{ }^{k}$ gives the steady state:

$$
s S=M_{0} \frac{(1-E B)+S_{1}\left(1-K_{1}^{n}\right) E B+K_{1}^{n} E B(1-E A)}{1+e f f . K_{1}^{n} E B E A}
$$

More precisely, we also have the convergence:

$$
\boldsymbol{M}_{z, \mathbf{4}}{ }^{\boldsymbol{k}}=s s+K^{k}\left(\boldsymbol{M}_{z, \mathbf{4}}^{\mathbf{0}}-s s\right), \quad K=-e f f . K_{1}^{n} E B E A
$$

\subsection{Modeling of an arbitrary MP-RAGE}

In the presence of multiple pulses and delays in the magnetization preparation, the ratio between magnetization before and after the preparation is no more constant. We assume that the action of the preparation on the magnetization can be modeled by a transfer matrix. We can write:

$$
M_{\text {out }}=\left(\begin{array}{cc}
A_{u} & q_{u} \\
0 & 1
\end{array}\right) M_{\text {In }}, M_{\text {out }}=U M_{\text {In }}
$$

$M_{\text {out }}$ : the magnetization after the preparation, $M_{I n}:$ the magnetization before the preparation. $U$ : the transfer matrix, which can be decomposed as the product of $N$ matrix, each modelling the action of a pulse followed by a time of relaxation. The magnetization vector is expressed along its homogeneous coordinates: $\left(M_{x}, M_{y}, M_{z}, 1\right) . u$ refers to the parameters of the preparation which contains for each RF pulse its amplitude, its phase and the delay before the next RF pulse. Considering that any transverse magnetization is adequately spoiled $(\mathrm{Mx}=\mathrm{My}=0)$, it follows that:

$$
\left(\begin{array}{c}
0 \\
0 \\
M_{z_{\text {out }}} \\
1
\end{array}\right)=\left(\begin{array}{cccc}
A_{u 1,1} & A_{u 1,2} & A_{u 1,3} & q_{u_{1}} \\
A_{u 2,1} & A_{u 2,2} & A_{u 2,3} & q_{u_{2}} \\
A_{u 3,1} & A_{u 3,2} & A_{u 3,3} & q_{u_{3}} \\
0 & 0 & 0 & 1
\end{array}\right)\left(\begin{array}{c}
0 \\
0 \\
M_{z_{\text {in }}} \\
1
\end{array}\right)
$$

We can retrieve the following transfer relation:

$M_{z \text { out }}=A_{u 3,3} M_{z \text { in }}+q_{u_{3}}$ i.e. $M_{z \text { out }}=f(u) M_{z \text { in }}+h(u)$

As $f(u)$ and $h(u)$ remain unchanged at each cycle, this allows updating the steady state (1) from the previous section:

$$
\begin{aligned}
\boldsymbol{M}_{z, \mathbf{4}}{ }^{\boldsymbol{k}+\mathbf{1}}=M_{0}(1 & -E B)+M_{0} S_{1}\left(1-K_{1}^{n}\right) E B \\
& +M_{0} K_{1}^{n} E B(1-E A) \\
& +K_{1}^{n} E B E A\left(f(u) \boldsymbol{M}_{\mathbf{z}, \mathbf{4}}{ }^{\boldsymbol{k}}+h(u)\right)
\end{aligned}
$$

$s s=$

$$
\frac{M_{0} \times\left[(1-E B)+S_{1}\left(1-K_{1}^{n}\right) E B+K_{1}^{n} E B(1-E A)\right]+K_{1}^{n} E B E A \boldsymbol{h}(\boldsymbol{u})}{1-K_{1}^{n} E B E A \boldsymbol{f}(\boldsymbol{u})}
$$

$$
s s(u)=\frac{A+\lambda \boldsymbol{h}(\boldsymbol{u})}{1-\lambda \boldsymbol{f}(\boldsymbol{u})}
$$

$A$ and $\lambda$ only depends on the acquisition parameters. $h(u)$ and $f(u)$ only depend on preparation parameters.

\subsection{Optimization of the magnetization preparation in the MP-RAGE}

The optimization of the preparation sequence requires the differentiation of the previously described steady state (2), with respect to $\mathbf{u}$ :

$$
\begin{array}{r}
\boldsymbol{\nabla s s}=(A+\lambda \boldsymbol{h}(\boldsymbol{u})) \frac{\lambda}{(1-\lambda \boldsymbol{f}(\boldsymbol{u}))^{2}} \cdot \boldsymbol{\nabla} \boldsymbol{f} \\
+\frac{\lambda}{1-\lambda \boldsymbol{f}(\boldsymbol{u})} \nabla \boldsymbol{h}(\boldsymbol{u})
\end{array}
$$

Recording that $f(u)=\vec{e}_{z} U \vec{e}_{z}$ and $h(u)=\vec{e}_{z} U \vec{e}_{0}$ where $\vec{e}_{z}=\left(\begin{array}{llll}0 & 0 & 1 & 0\end{array}\right)$ and $\vec{e}_{0}=\left(\begin{array}{llll}0 & 0 & 0 & 1\end{array}\right)$, the transfer matrix is :

$$
U=\left(\begin{array}{cccc}
* & * & * & * \\
* & * & * & * \\
* & * & f(u) & h(u) \\
0 & 0 & 0 & 1
\end{array}\right)
$$

For an arbitrary $\mathrm{N}$-pulse preparation: $U=U_{N} \ldots U_{2} U_{1}$. $U_{i}=U\left(u_{i}\right)$ is the transfer matrix modeling the action of $i$ th pulse and delay whose parameters are regrouped into the vector $u_{i}$. So:

$$
\underset{u_{i}}{\nabla} f=\vec{e}_{z} \frac{\partial U}{\partial u_{i}} \vec{e}_{z} \quad \underset{u_{i}}{\nabla} g=\vec{e}_{z} \frac{\partial U}{\partial u_{i}} \vec{e}_{0}
$$

At this point, we have expressed the steady state and its partial derivatives with respect to the preparation scheme parameters. The standard GRAPE algorithm can thus be used: with the chain rule derivation, we can derivate any cost functions that are composite functions of $s s(u)$. The algorithm is coded on Matlab (The MathWorks, Inc., Natick, $M A, U S A, R 2019 b)$. A second order gradient descent is performed thanks to the Matlab function fmincon, to which gradient expressions derived in the previous equations are provided. 


\subsection{Numerical simulation to enhance contrast between gray and white matter}

To validate the proposed method, we have compared our numerical results with those of Mugler and al. They optimized a preparation composed of a single non-selective radiofrequency pulse with flip angle between $0^{\circ}$ and $180^{\circ}$. In their model, 32-step phase encoding is performed at each cycle with TR/TE of $10 / 4 \mathrm{~ms}$. The goal is to optimize the signal intensity between white matter $\left(T_{1} / T_{2} /\right.$ proton density: $550 / 90 \mathrm{~ms} / 0.65)$ and gray matter $\left(\mathrm{T}_{1} / \mathrm{T}_{2} /\right.$ proton density: $940 / 100 \mathrm{~ms} / 0.75$ ). There is no recovery between the end of the acquisition and the preparation pulse. The time between the pulse and the beginning of the acquisition is fixed to $140 \mathrm{~ms}$ to fulfill the constraint of acquisition time. They have found for each flip angle of the gradient-echo acquisition the best angle for preparation pulse to maximize white matter and gray matter signal difference (WGSD). For each flip angle used in the gradient-echo, they plotted the difference between the maximum WGSD obtained with the optimal preparation pulse and the WGSD without any preparation. To retrieve this result, we search the minimum of the following cost function thanks to our adaptation of GRAPE:

$$
C=-\left|S_{W M}\right|+\left|S_{G M}\right|=-W G S D
$$

$S_{W M}$ and $S_{G M}$ are respectively the signal intensities of the white and gray matter at the center of the k-space containing $n$ lines.

$S=\left[s+\left(\frac{s s(u)-(1-E B)}{E B}-s\right) K_{1}^{-p}\right] \sin (\alpha) e^{-\frac{T E}{T 2}}$

With $s=M_{0} S_{1}, \quad p=\frac{n}{2}$ or $n$ for respectively centrally reordered or sequential phase encoding. Due to absolute value, the function of (3) is not differentiable in some points, which can lead to errors. In this specific case, the parametrization used in the optimization ensures to be in a domain where the gradient is defined. The computation of the gradient of the cost function is based on paragraph $\mathbf{2 . 3}$ and general rules of differentiation.

\subsection{Maximization of the contrast between cortex and corpus callosum}

On the assumption that exploiting $T_{1}$ and $T_{2}$ may enhance contrast, we used our algorithm to maximize contrast between cortex and corpus callosum on a rat brain at 11.7T. We consider that the best cost function to enhance contrast between two tissues aims at saturating the signal from one tissue while maximizing the signal from the other. Thus, we chose the following cost function:

$$
C=\alpha \cdot S_{1}{ }^{2}-\operatorname{sign}\left(S_{2}\right) S_{2}{ }^{2}
$$

sign : is added to force solution with positive value for the signal of species 2 in the center of the k-space. $\alpha$ : a weighting coefficient (10 in our case).

We implemented the solution on a preclinical 11.7T scanner (Bruker). The objective was to optimize the best parameters to saturate the cortex $\left(\mathrm{T}_{1} / \mathrm{T}_{2}: 1945.0 / 34.2 \mathrm{~ms}\right)$ and maximize the signal of corpus callosum ( $\left.\mathrm{T}_{1} / \mathrm{T}_{2}: 1710 / 31.2 \mathrm{~ms}\right) . \mathrm{T}_{1}$ and $\mathrm{T}_{2}$ values have been estimated on the basis of the values measured by de Graaf and al. [7] and the application manual of the constructor. We considered in a first approximation the proton densities for both tissues as equal to 1 . The duration of the sequence was constant. Main parameters of the sequence are summarized in the following table:

\begin{tabular}{|l|l|}
\hline Number of lines per cycle & 64 \\
\hline TR/TE/Angle of excitation & $6.5 \mathrm{~ms} / 2.2 \mathrm{~ms} / 13^{\circ}$ \\
\hline Time of a cycle & $3000 \mathrm{~ms}$ \\
\hline Number of accumulations & 8 \\
\hline Time of acquisition & $438 \mathrm{~s}$ \\
\hline Phase encoding & sequential \\
\hline FOV/slice thickness & $35 \times 35 \mathrm{~mm} / 6 \mathrm{~mm}$ \\
\hline Matrix size & $128 \times 128 \times 8$ \\
\hline
\end{tabular}

Table. 1. Acquisition parameters

\section{RESULTS}

\subsection{Numerical simulation to enhance contrast between gray and white matter: results}

The results below obtained with our optimization well agree with the previous results of Mugler and al. [3]. This, somehow, validates the efficiency of the proposed approach for a relatively simple optimization problem.

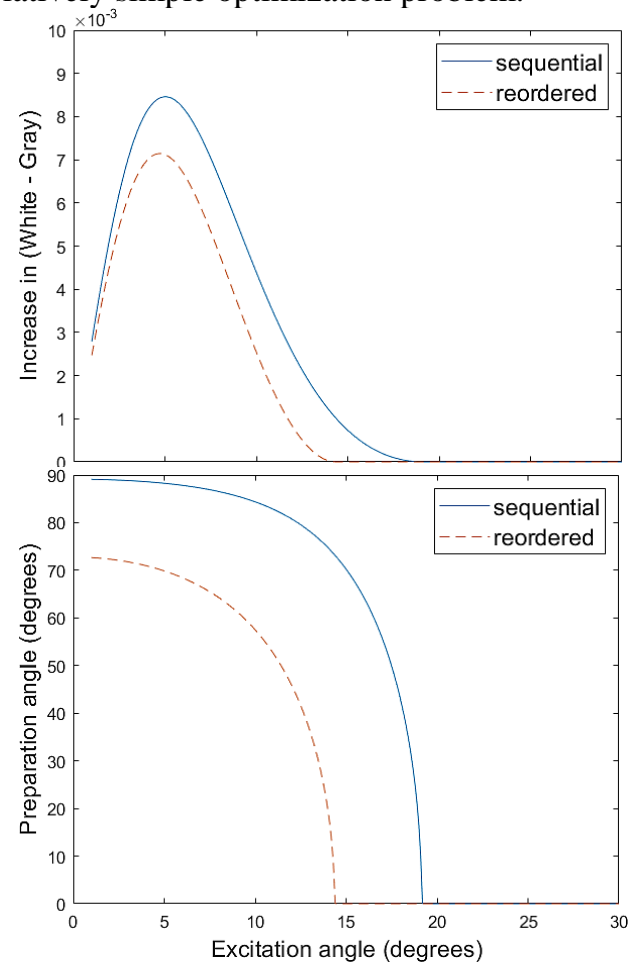


Fig. 2. In the top graph, maximum WGSD minus WGSD without preparation as a function of the flip angle of the gradient echo acquisition. The ordinate in units of normalized transverse magnetization. In the bottom graph, preparation flip angle yielding the maximum WGSD as a function of the flip angle of the gradient echo acquisition.

\subsection{Maximization of the contrast between cortex and corpus callosum: in vivo results}

Considering the optimization of a single-pulse preparation, the optimal solution is a standard MP-RAGE with an inversion pulse $\left(180^{\circ}\right)$ followed by an inversion time of 925 $\mathrm{ms}$ (from the pulse to the first excitation).

Considering the optimization of a two-pulse preparation, the optimal solution is $90^{\circ}-17 \mathrm{~ms}-90^{\circ}-646 \mathrm{~ms}$ - first excitation. In order to counter the effect of B0 inhomogeneities, which are not taken into account in the optimization, a refocusing pulse is inserted between the two pulses. The final preparation results in an optimized $\mathrm{T}_{2}$ Prep-IR.

In both case, we numerically find a similar contrast of $0,93\left(C=\frac{I_{\max }-I_{\min }}{I_{\max }+I_{\min }}\right)$ and a signal $10 \%$ higher with the two-pulse preparation.

In vivo, we verify the saturation of the cortex and the enhancement of the corpus callosum (Fig. 3.). As expected,

the $T_{2}$ Prep-IR solution gives a higher signal -to-noise ratio. This is explained by the fact that the proposed scheme uses $\mathrm{T}_{2}$ relaxation (in addition to $\mathrm{T}_{1}$ ) to generate contrast, which is inherently faster than using only $\mathrm{T}_{1}$. For a similar contrast to IR, $T_{2}$ Prep-IR is shorter. This .provides more time to the magnetization recovery before the preparation and so a better signal dynamics.

\begin{tabular}{lllll} 
& \multicolumn{2}{l}{$\begin{array}{l}\text { Intensity mean } \pm \text { standard } \\
\text { deviation }\end{array}$} & $\begin{array}{l}\text { Contrast } \\
\text { to noise : }\end{array}$ \\
& $\mathrm{CC}^{1}:$ & $\mathrm{CX}^{2}:$ & Noise : & $\frac{I_{\text {max }}-_{\min }}{\text { Noise }}$ \\
TI & $1610 \pm 467$ & $710 \pm 329$ & $359 \pm 93$ & 2,5 \\
$\mathrm{~T}_{2}$ Prep- & $2441 \pm 492$ & $978 \pm 422$ & $363 \pm 95$ & 4,03 \\
IR & & & &
\end{tabular}

1: corpus callosum, 2: cortex.

Table. 2. Intensity and Contrast-to-noise ratio in vivo

\section{CONCLUSION}

We have developed a new tool based on the GRAPE algorithm to optimize an arbitrary preparation in a MPRAGE, with the aim to improve contrast between two target tissues. We validated our approach by numerical results and in vivo acquisitions. In the in vivo application, the resulting preparation sequence is an optimized $\mathrm{T}_{2}$ prep-IR, which significantly improves both signal and contrast-to-noise ratio compared to the standard IR strategy.

The novelty of our algorithm with respect to previous optimal control algorithms is the inclusion of the steady state in the optimization. Our framework is general and could be applied to complex contrast problems, particularly involving more than two targeted tissues. In addition, the proposed framework could be applied to other rapid MRI sequences, with some adaptations that are currently under consideration.

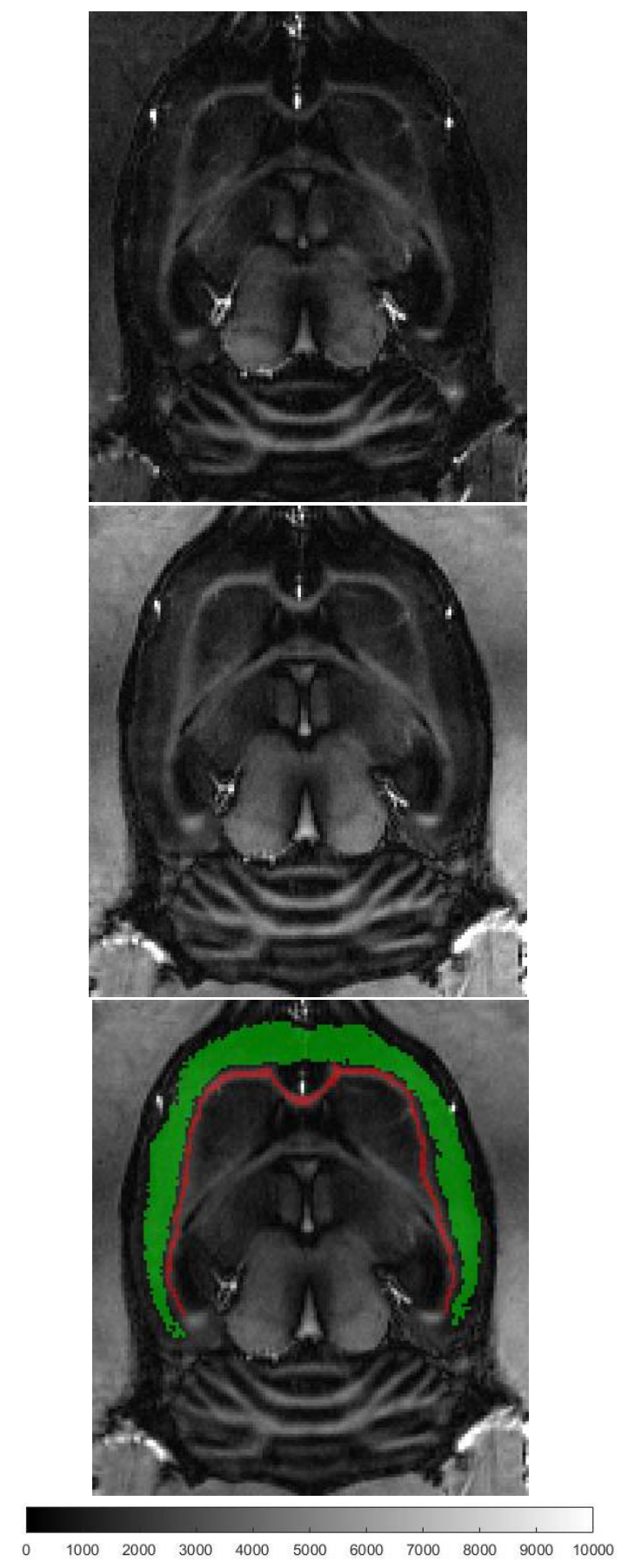

Fig. 3. In the top image: Standard MP-RAGE with inversion recovery ( $T I=925 \mathrm{~ms})$ aiming at saturating the cortex and maximizing the corpus callosum (results of one pulse optimization as described below). In the middle image: MP-RAGE with $\mathrm{T}_{2}$ Prep-IR aiming at saturating the cortex and maximizing the corpus callosum (results of two pulses optimization as described below). In the bottom image: segmented cortex (green) and segmented corpus callosum (red). 


\section{ACKNOWLEDGEMENTS}

This work was performed on the platform PILoT, member of France Life Imaging network (grant ANR-11-INBS-

0006) and within the framework of LABEX PRIMES ANR-

\section{1-LABX-0063/ ANR-11-IDEX-0007.}

\section{REFERENCES}

[1] J. P. Mugler and J. R. Brookeman, "Three-dimensional magnetization-prepared rapid gradient-echo imaging (3D MP RAGE)," Magnetic Resonance in Medicine, vol. 15, no. 1, pp. 152-157, 1990, doi: 10.1002/mrm.1910150117.

[2] F. H. Epstein, J. P. Mugler, and J. R. Brookeman, "Spoiling of transverse magnetization in gradient-echo (GRE) imaging during the approach to steady state," Magn. Reson. Med., vol. 35, no. 2, pp. 237-245, Feb. 1996, doi: $10.1002 / \mathrm{mrm} .1910350216$.

[3] Mugler JP 3rd,Brookeman JR, "Rapid three-dimensional T1weighted MR imaging with the MP-RAGE sequence," 1991.

[4] J. P. Marques, "MP2RAGE, a self bias-field corrected sequence for improved segmentation and T1-mapping at high field."

[5] N. Khaneja, T. Reiss, C. Kehlet, T. Schulte-Herbrüggen, and S. J. Glaser, "Optimal control of coupled spin dynamics: design of NMR pulse sequences by gradient ascent algorithms," Journal of Magnetic Resonance, vol. 172, no. 2, pp. 296-305, Feb. 2005, doi: 10.1016/j.jmr.2004.11.004.

[6] E. Van Reeth, H. Ratiney, M. Lapert, S. J. Glaser, and D. Sugny, "Optimal control theory for applications in Magnetic Resonance Imaging,” Pac. J. Math. Ind., vol. 9, no. 1, p. 9, Dec. 2017, doi: 10.1186/s40736-017-0034-3.

[7] de Graaf et al., "High Magnetic Field Water and Metabolite Proton T1 and T2 Relaxation in Rat Brain In Vivo," Magnetic Resonance in Medicine", vol 56, no. 2, June 2006, pp. 386394, doi: 10.1002/mrm.20946.

\section{COMPLIANCE WITH ETHICAL STANDARDS}

In vivo studies were conducted in accordance with ethical compliance (agreement C69266 1401). 